\title{
Evaluation De La Qualité Microbiologique Des Médicaments Traditionnels Améliorés (MTA) Vendus Dans Six Communes d'Abidjan (Côte d'Ivoire)
}

\section{Coulibaly Bakary}

Maître-Assistant, Enseignant-Chercheur, Laboratoire d'Agrovalorisation, Département de Biochimie-Microbiologie, UFR Agroforesterie, Université Jean Lorougnon Guédé, Daloa, Côte d'Ivoire

\section{Kouassi Kouassi Clément}

Maître-Assistant, Enseignant-Chercheur, Laboratoire d'Agrovalorisation,

Département de Biochimie-Microbiologie, UFR Agroforesterie,

Université Jean Lorougnon Guédé, Daloa, Côte d'Ivoire

\section{Kroa Ehoulé}

Chargé de recherche, Médecin, Programme National de Promotion de la

Médecine Traditionnelle, Ministère de la Santé et de la lutte contre le SIDA, Côte d'Ivoire

\section{Konaté Ibrahim}

Maître de Conférences, Enseignant-Chercheur, Laboratoire d'Agrovalorisation,

Département de Biochimie-Microbiologie, UFR Agroforesterie, Université Jean Lorougnon Guédé, Daloa, Côte d'Ivoire

\section{Kouassi Kporou Elisée}

Maître-Assistant, Enseignant-Chercheur, Laboratoire d'Agrovalorisation,

Département de Biochimie-Microbiologie, UFR Agroforesterie,

Université Jean Lorougnon Guédé, Daloa, Côte d'Ivoire

\section{Djaman Allico Joseph}

Professeur titulaire, Enseignant-Chercheur, Laboratoire de

Pharmacodynamie-Biochimique, UFR Biosciences,

Université Félix Houphouët-Boigny, Cocody, Abidjan, Côte d'Ivoire

Doi: 10.19044/esj.2018.v14n15p307 URL:http://dx.doi.org/10.19044/esj.2018.v14n15p307

\begin{abstract}
This study was carried out in six (6) communes of Abidjan within a period of six months (November 2016 to April 2017). This paper focuses on assessing the microbiological quality of traditional medicines produced and sold in these communes. A total of 38 samples were collected, including 19 in
\end{abstract}


powder form and another 19 in liquid form. The search and enumeration of the germs was carried out according to the method based on standard procedures (AFNOR). Enumeration included total mesophilic aerobic flora, total coliforms, yeasts and molds, Staphylococcus aureus, and salmonella. The results indicate an absence of the pathogenic flora (Salmonella and S. aureus). There is also an absence of yeasts and molds in the products in liquid form. The level of contamination of the Mesophilic Total Aerobic Flora (FMAT) ranges from 1.104 to 6.7.107 CFU / $\mathrm{ml}$ or CFU / $\mathrm{g}$ and from 1.6.104 to 5.5.107 $\mathrm{CFU} / \mathrm{g}$ or CFU / $\mathrm{ml}$ for total coliforms. In terms of microbiological quality, $39.47 \%$ of the analyzed samples had compliant. In view of these results, it is necessary to train Traditional Medicine Practitioners on Good Manufacturing Practices (GMP) methods throughout the production chain in order to market MTAs of guaranteed quality and safety.

Keywords: Traditional Improved Medicines (MTA), Microbiological Quality, Traditional Medicine Practitioners (PMT), S. aureus, Coliforms

\section{Résumé}

Le présent travail a été réalisé dans six (06) communes d'Abidjan durant une période de six mois. Il visait à apprécier la qualité microbiologique des médicaments traditionnels produits et vendus dans ces communes. Au total, 38 échantillons ont été collectés dont 19 en poudre et 19 en liquide. La recherche et le dénombrement des germes ont été effectué selon la méthode basée sur les procédures normalisées (AFNOR). Le dénombrement a porté sur la flore aérobie mésophile totale, les coliformes totaux, les levures et moisissures, la recherche de Staphylococcus aureus, et des salmonelles. Les résultats indiquent une absence de la flore pathogène (les Salmonelles et $S$. aureus). On note également une absence des levures et des moisissures dans les produits sous la forme liquide. Le niveau de contamination de la Flore Mésophile Aérobie Totale (FMAT) varie de $1,1.10^{4}$ à $6,7.10^{7} \mathrm{UFC} / \mathrm{ml}$ ou (UFC/g) et de $1,6.10^{4}$ à $5,5.10^{7} \mathrm{UFC} / \mathrm{g}$ ou $(\mathrm{UFC} / \mathrm{ml})$ pour les Coliformes Totaux. Au vu de ces résultats, il est nécessaire de former les Praticiens de Médecine traditionnelle sur les méthodes de Bonnes Pratiques de Fabrication au cours de la chaîne de production afin de mettre sur le marché des Médicaments Traditionnels Améliorés (MTA) de qualité et d'innocuité garanties.

Mots-clés: Médicaments Traditionnels Améliorés (MTA), qualité microbiologique, Praticiens de Médecine traditionnelle (PMT), S. aureus, Coliformes 


\section{Introduction}

Les médicaments à base de plantes, sont très utilisés et ont une importance considérable dans le commerce international (OMS, 1998 ; OMS, 2000; Coulibaly, 2009). Ainsi, l'Organisation Mondiale de la Santé (OMS) estime que $80 \%$ de la population africaine a toujours recours à la Médecine Traditionnelle (MT) pour les soins de santé primaire (OMS, 2002).

Aussi, l'Union Africaine a exprimé un intérêt réel pour sa promotion et sa valorisation lors de la première conférence portant sur les plantes médicinales et la pharmacopée africaine tenue à Dakar (Sénégal) (Abayomi, 1996).

En Côte d'Ivoire, après avoir été pendant longtemps le souci des seuls chercheurs, la Médecine traditionnelle est devenue aujourd'hui une composante de la politique sanitaire.

En août 1995, le Ministère de la Santé et de l'Hygiène Publique a intégré cette Médecine dans son Plan National de Développement Sanitaire (PNDS) (Konan, 2012). Vu, la place qu'occupent les médicaments traditionnels dans le programme de santé publique, les autorités sanitaires et la population accordent de l'importance à l'innocuité, à l'efficacité ainsi qu'à la qualité de ces médicaments à base de plantes. La contamination des médicaments à base de plantes affecte la qualité de ceux-ci (Becila, 2009). Elle peut être aussi à l'origine d'une altération du médicament, lui faisant perdre ses caractéristiques organoleptiques et/ou commerciales, parfois être la cause d'intoxications ou toxi-infections graves. Ainsi, pour assurer une bonne commercialisation de ces médicaments à base de plante, l'évaluation de la qualité microbiologique est nécessaire afin d'apprécier le niveau de contamination de ces médicaments et déterminer le risque que ceux-ci pourraient présenter sur la population. L'objectif principal de cette étude est d'évaluer la qualité microbiologique des médicaments à base de plantes produits par des Praticiens de Médecine Traditionnelle (PMT) de six communes d'Abidjan.

Le travail consiste à recenser les Médicaments Traditionnels Améliorés (MTA) produits par les PMT dans les Centres de Médecine traditionnelle (CMT) ou unités de production; à déterminer les microorganismes susceptibles d'altérer la qualité marchande et organoleptique ; à dénombrer les germes présents et à rechercher les germes pathogène. 


\section{Materiel et methodes}

\section{Materiel}

\section{Matériel biologique}

Il se compose de médicaments à base de plantes (poudre ou liquide) produits par les Praticiens de Médecine Traditionnelle référés par le Programme National de Promotion de la Médecine Traditionnelle

\section{Echantillonnage}

\section{Taille de l'échantillonnage}

$\mathrm{Au}$ total, 38 échantillons de médicaments traditionnels améliorés ont été collectés auprès de 20 praticiens de médecine traditionnelle dont 19 médicaments traditionnels sous la forme liquide et 19 autres en poudre.

\section{Methodes}

\section{Analyses microbiologiques}

La recherche et le dénombrement des germes ont été effectué selon la méthode basée sur les procédures normalisées (AFNOR, 1996). Le dénombrement a porté sur la flore aérobie mésophile totale (NF.V08-51), les coliformes totaux (NF.V08-50), les levures et moisissures (Normes XP-V08059-oct 1996), la recherche de Staphylococcus aureus (NF.V08-57), et des salmonelles (NF.V08-052, 1997).

\section{Méthode de préparation des milieux de cultures}

Les milieux de cultures ont été préparés selon les recommandations des fabricants.

\section{Préparation des échantillons}

Une solution mère a été préparée. Ainsi $25 \mathrm{~g}$ ou $25 \mathrm{~mL}$ de la solution à analyser ont été prélevée de façon aseptique et ajouté à $225 \mathrm{~mL}$ d'Eau Peptonée tamponnée (EPT) stérile et homogénéisé pendant $2 \mathrm{~min}$. La solution obtenue a été appelé solution mère (SM).

Pour la dilution, $1 \mathrm{~mL}$ de la $\mathrm{SM}$ est prélevé et ajouté à $9 \mathrm{~mL}$ d'eau distillée stérile afin d'avoir une dilution $10^{-1}$. $1 \mathrm{~mL}$ de la dilution $10^{-1}$ est prélevé à nouveau et ajouté à $9 \mathrm{~mL}$ d'eau distillée stérile pour obtenir la dilution $10^{-2}$. Cette opération est répétée jusqu'à obtenir une solution de dilution $10^{-4}$ (AFNOR, 1996) 
Ensemencement des milieux de cultures pour recherche et dénombrement des germes)

Ensemencement en surface (milieux PCA, VRBG, Sabouraud + Chloramphénicol, Baird Parker)

$0,1 \mathrm{~mL}$ de chaque dilution $\left(10^{-1}, 10^{-2}, 10^{-3}\right.$ et $\left.10^{-4}\right)$ est prélevée puis déposée aseptiquement à la surface de la gélose contenue dans la boîte de Pétri. Ensuite à l'aide d'un râteau stérile, un étalement uniforme à la surface de la boîte de Pétri est réalisée. Les boites ont été incubées à $30^{\circ} \mathrm{C}$ et $37^{\circ} \mathrm{C}$ puis observés après $24 \mathrm{H}$.

\section{Ensemencement en profondeur (milieux PCA, VRBG)}

$1 \mathrm{~mL}$ de chaque dilution $\left(10^{-1}, 10^{-2}, 10^{-3}\right.$ et $\left.10^{-4}\right)$ a été transféré aseptiquement dans les boîtes de Pétri stérile correspondante. Le milieu a été refroidi au bain-marie à $45^{\circ} \mathrm{C}$ et ajouté à l'échantillon à raison de $15 \mathrm{~mL}$ par boîte. Ensuite, des mouvements rotatifs de la boîte de Pétri ont été réalisé afin d'avoir une répartition homogène de l'échantillon dans la boîte de Pétri. La boîte est par la suite fermée puis laissée au repos sur une surface parfaitement horizontale jusqu'à solidification complète de la première couche. Après la solidification, une deuxième couche de $7 \mathrm{~mL}$ de milieu est ajoutée pour une nouvelle solidification. Après cette seconde solidification les boîtes sont retournées. Les boites ont été incubées à $30^{\circ} \mathrm{C}$ et $37^{\circ} \mathrm{C}$ puis observés après $24 \mathrm{H}$.

\section{Ensemencement du milieu SS pour isolement des salmonelles}

$0,1 \mathrm{~mL}$ de chaque dilution $\left(10^{-1}, 10^{-2}, 10^{-3}\right.$ et $\left.10^{-4}\right)$ a été transféré aseptiquement dans les tubes contenant $10 \mathrm{~mL}$ du milieu Rappaport Vassiliadis (RV) correspondante. Les tubes ont été incubés à $37^{\circ} \mathrm{C}$ puis observés après $24 \mathrm{H}$. Ensuite à l'aide d'une anse de platine stérile une quantité du bouillon est prélevé puis ensemencé sous forme de stries sur le milieu SS. Les boites sont incubées à $37^{\circ} \mathrm{C}$ puis observés après $24 \mathrm{H}$.

\section{Comptage des colonies}

Selon la norme AFNOR (1996), ont été retenues les boîtes ayant plus de 30 et moins de 300 colonies. Le nombre de microorganismes présents dans un échantillon de produit donné s'obtient par la formule suivante selon la norme AFNOR (2001).

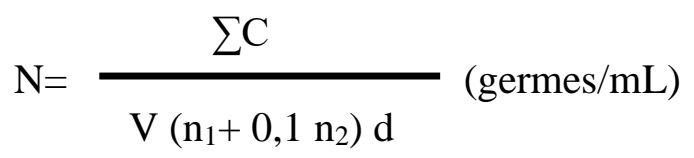

$\mathrm{N}$ : nombres de germes 
$\sum \mathrm{C}:$ sommes des colonies

$\mathrm{V}$ : volume de l'inoculum

$\mathrm{n}_{1}$ : nombres de boites de Pétri comptées à la $1^{\text {ère }}$ dilution

$\mathrm{n}_{2}$ : nombres de boites de Pétri comptées à la $2^{\text {ème }}$ dilution

$\mathrm{d}$ : taux de dilution à la première dilution retenue

\section{Normes microbiologiques}

Le nombre de microorganisme par $\mathrm{mL}$ ou g a été calculé pour chaque flore étudiée en fonction des échantillons analysés de chaque commune, puis comparé à la référence normative des critères microbiologiques des médicaments à base de plantes de la pharmacopée européenne (Ph EU 8.0, 2014).

\section{Resultats et discussion}

\section{Analyses microbiologiques}

Cinq (05) germes (FMAT (Flore Mésophile Aérobie Totale), coliformes totaux, levures moisissures, salmonelles, $S$. aureus) ont été recherchés dans chaque échantillon.

La FMAT est absente dans respectivement $21,05 \%$ des échantillons en poudre et $31,57 \%$ des échantillons liquide. Par ailleurs, 13 échantillons sur 19 de MTA en poudre et 11 échantillons sur 19 de MTA en liquide ont une teneur en FMAT comprise entre 0 et $10^{7} \mathrm{ufc} / \mathrm{g}$ soit respectivement des taux de $68,42 \%$ et 57,89\%. Par contre, 2 échantillons des 19 échantillons de MTA en poudre et 2 échantillons des MTA en liquide ont une teneur en FMAT supérieure à $10^{7} \mathrm{ufc} / \mathrm{g}$. Ainsi, la FMAT est présente dans 28 échantillons sur les 38 analysés. Les concentrations varient dans l'ordre de 0 à plus de $6,7.10^{7}$ ufc/g (Tableau I).

La flore totale renseigne les conditions de préparation, l'efficacité des procédés de traitement et de conservation. Selon les normes (AFNOR, 1996), les échantillons les plus contaminés sont ceux qui présentent un taux de flore totale supérieur à $10^{7}$ germes $/ \mathrm{g}$. Ils sont considérés comme non satisfaisants. Les résultats des analyses montrent que la flore totale est présente dans 10,52\% des MTA liquides et des MTA en poudre avec un niveau de contamination supérieur à $10^{7} \mathrm{ufc} / \mathrm{g}$ donc de qualité microbiologique insatisfaisants. Ce résultat est supérieur à celui trouvé par Diabaté (1991) avec $0 \%$; Alassane (1988) avec 2,27 \% et Sylla (2000) avec $4 \%$. Ceci s'expliquerait par une contamination due à la mauvaise conservation des produits, mais aussi le manque d'unité de production agrée pour les PMT. Ainsi, des efforts restent à faire en matière d'application des Bonnes Pratiques de Fabrication (BPF). Car la présence de cette flore à un taux aussi élevé témoigne du non-respect total des Bonnes pratiques de fabrication (BPF). 
Tableau I. Niveau de contamination des MTA par la Flore Mésophile Aérobie Totale (FMAT)

\begin{tabular}{cccc}
\hline Produits & Contamination & Prélèvements & Pourcentage(\%) \\
\hline \multirow{2}{*}{ MTA en poudre } & Absence & 04 & $21,05 \%$ \\
& $0<\mathrm{F}<10^{7}$ & 13 & $68,42 \%$ \\
& $\mathrm{~F}>10^{7}$ & 02 & $10,52 \%$ \\
\hline \multirow{2}{*}{ MTA liquide } & Absence & 06 & $31,57 \%$ \\
& $0<\mathrm{F}<10^{7}$ & 11 & $57,89 \%$ \\
\hline
\end{tabular}

Les coliformes totaux sont présents dans 22 échantillons analysés soit $57,84 \%$. Les résultats du Tableau II indiquent également une absence de ces coliformes dans 12 MTA en poudre et 4 MTA en liquide. Ils ont une teneur supérieure à $10^{7} \mathrm{UFC} / \mathrm{g}$ dans 7 des échantillons en poudre et 15 des échantillons liquides. Les résultats montrent que 36,84 \% des produits analysés ont un taux supérieur à $10^{7} \mathrm{UFC} / \mathrm{g}$ au niveau des MTA en poudre et un taux de 78,94\% pour les échantillons liquide. Cette valeur est largement au-dessus de la norme de la pharmacopée européenne $10^{3}$ valeur maximale au-delà de laquelle les produits sont impropres à l'utilisation humaine (Lehmann, 2013 ; Ph EU 8.0 2014). En, somme 57,89 \% des échantillons sont non satisfaisants. Ces résultats sont comparables à ceux d'Alassane (1988) trouvés dans une étude similaire, $35,71 \%$ de coliformes totaux. Ces valeurs sont largement supérieures à celles trouvé par Sylla (2000), avec $9 \%$ et Diabaté (1991) avec 18,64\%. La forte fréquence des coliformes totaux témoigne d'une contamination fécale. Ceci s'expliquerait par l'absence de contrôle des règles d'hygiène et de conservation des produits finis.

Tableau II. Niveau de contamination des MTA par les Coliformes totaux

\begin{tabular}{cccc}
\hline Produits & Contamination & Prélèvements & Pourcentage(\%) \\
\hline \multirow{2}{*}{ Absence } & 12 & $63,15 \%$ \\
$0<\mathrm{F}<10^{7}$ & 00 & $0 \%$ \\
MTA en poudre & $\mathrm{F}>10^{7}$ & 07 & $36,84 \%$ \\
& Absence & 04 & $21,05 \%$ \\
& $0<\mathrm{F}<10^{7}$ & 00 & $0 \%$ \\
& $\mathrm{~F}>10^{7}$ & 15 & $78,94 \%$ \\
\hline
\end{tabular}


Le Tableau III révèle une absence des levures et moisissures dans $21,05 \%$ des échantillons en poudre et une absence totale dans les échantillons liquides. Neuf échantillons sur 19 échantillons de MTA en poudre ont une teneur en flore fongique comprise entre 0 et $10^{7} \mathrm{UFC} / \mathrm{g}$ soit $47,36 \%$. En outre, il est noté une teneur en levures et moisissures supérieur à $10^{7} \mathrm{UFC} / \mathrm{g}$ dans $31,57 \%$ des échantillons. Les levures et moisissures présentent une fréquence variable de 0 à $7,8.10^{7} \mathrm{UFC} / \mathrm{g}$. Les résultats révèlent que 15 MTA sont contaminés par les germes fongiques soit $39,47 \%$. La forte présence de cette flore est sans doute due à la durée de stockage du produit en poudre Bonfoh (2002). Dieng (2001), a trouvé, pour les mêmes types de produit en poudre, une contamination de $51 \%$. Les levures et moisissures observées dans les produits proviendraient des matières premières ou du milieu ambiant (Tayou, 2007).

Tableau III. Niveau de contamination des MTA par les levures et moisissures

\begin{tabular}{cccc}
\hline Produits & Contamination & Prélèvements & Pourcentage $(\%)$ \\
\hline \multirow{2}{*}{ MTA en poudre } & $0<\mathrm{F}<10^{7}$ & 04 & $21,05 \%$ \\
& $\mathrm{~F}>10^{7}$ & 09 & $47,36 \%$ \\
Absence & 06 & $31,57 \%$ \\
MTA liquide & $0<\mathrm{F}<10^{7}$ & 19 & $100 \%$ \\
& $\mathrm{~F}>10^{7}$ & 00 & $0 \%$ \\
\hline
\end{tabular}

Les résultats de cette étude indiquent également une absence totale de bactéries pathogènes que sont: $\mathrm{S}$. aureus et les salmonelles.

Aucun $S$. aureus n'a été mis en évidence, donc le taux de conformité est de $100 \%$ recherchés dans les produits naturels Cissé (1991).

Aucune salmonelle n'a été mise également en évidence. Mais la fréquence élevée des coliformes totaux indique une forte suspicion étant donné que la survie de coliformes fécaux dans l'environnement est semblable à celle des salmonelles. L'absence des staphylocoques pathogènes dans les échantillons peut s'expliquer par leur inhibition par d'autres microorganismes Fleurette (1991). L'absence de salmonelles pourrait s'expliquer également par l'inefficacité des méthodes de recherche classiques utilisées. Ces résultats sont conformes à ceux de Catsaras et al. (1984). Ces auteurs ont montré que la recherche des salmonelles par la méthode classique peut être négative bien que l'échantillon en renferme $10^{5}$ à $10^{8}$ germes $/ g$ ou germes $/ \mathrm{ml}$. Ce fait serait lié selon eux à la présence de germes inhibiteurs (coliformes). Ces résultats confirment ceux de Dione (2000) et Njassap (2001). Ces auteurs ont noté une absence de salmonelles dans les produits analysés. 


\section{Conclusion}

Un médicament à base de plante est un produit dont la composition possède des propriétés curatives et préventives. Il ne peut être mis sur le marché qu'à l'issue des contrôles de la qualité portant sur toute la chaine de production. Ainsi, 38 produits à base de plantes dont 19 en poudre et 19 en liquide ont fait 1'objet de cette étude. L'analyse microbiologique a porté sur 5 germes différents. Les résultats microbiologiques obtenus sont très variables en fonction des germes recherchés. Ces résultats révèlent que la plupart des échantillons sont contaminés par la FMAT au-delà du seuil d'acceptabilité. Parallèlement, une forte présence de coliformes totaux révèle que 57,84 \% des échantillons sont impropres à la consommation humaine. Par contre, aucune levure et moisissure n'ont été mises en évidence dans les produits liquides analysés. On note une absence de germes pathogènes dans les échantillons de MTA analysés. Toutefois, l'ensemble des PMT doit adopter une démarche qualité dans leur système de production afin de garantir la qualité et son efficacité.

\section{References:}

1. Abayomi, S. (1996). Plantes médicinales et Médecine Traditionnelle d'Afrique, Paris, Editions Karthala, 375 p.

2. AFNOR (1996). Microbiologie des aliments - Dénombrement des coliformes thermotolérants par comptage de colonies obtenues à $44^{\circ} \mathrm{C}$, NF V 08 60, Paris, 20p

3. Alassane, A. (1988). Contribution à l'étude de l'hygiène dans la restauration collective au centre des Euvres universitaires de Dakar (C.O.U.D). Thèse Médecine Vétérinaire. Sénégal n²6, 213p.

4. Becila, A. (2009). Préventions des altérations et des contaminations microbiennes des aliments. Mémoire post-Graduation Spécialisée, Institut de la nutrition, de l'alimentation et des technologies agroalimentaires (INATAA), Université Mentouri-Constantine, Algérie 90 p.

5. Bonfoh, B. (2002). Qualité microbiologique des produits en poudre vendus en saison chaude dans le district de Bamako au Mali. (www.laitsain.com).

6. Catsaras, M. \& Grebot, D. (1984). Multiplication des salmonelles dans la viande hachée. Bulletin Académique Vétérinaire, 57 : 501-502.

7. Coulibaly, S.L. (2009). Contribution à l'évaluation de la qualité des médicaments traditionnels améliorés. Thèse de pharmacie de l’Université de Bamako, Mali,: 80 p.

8. Diabaté, V. (1991). Contribution à l'étude de l'hygiène de la restauration collective en Côte d'Ivoire : Cas du Centre Hospitalier Universitaire (CHU) de COCODY d'Abidjan. Thèse Médecine 
Vétérinaire. Dakar, 196p.

9. Dione, A. (2000). Contribution à l'étude de la qualité bactériologique de quelques denrées alimentaires d'origine animale commercialisées sur le marché dakarois : Thèse de Médecine Vétérinaire, Dakar, 03, $212 \mathrm{p}$.

10. Dieng, M. (2001). Contribution à l'étude de la qualité microbiologique des laits caillés industriels commercialisés sur les marchés dakarois. Thèse de Médecine Vétérinaire, Dakar, 210p.

11. Fleurette, J. (1991). Intoxications alimentaires à Staphylocoques. Le Point sur Paris,: pp 23 - 25.

12. Konan, A. (2012). Place de la Médecine Traditionnelle dans les soins de santé primaires à Abidjan (Côte d'Ivoire).Faculté de médecine. Thèse de doctorat de l'Université de TOULOUSE III-PAUL SABATIER, $104 \mathrm{p}$.

13. Lehmann, H. (2013). Le médicament à base de plantes en Europe. Statut, enregistrement, contrôles. Thèse de doctorat de l'Université de Strasbourg. Faculté de Pharmacie Laboratoire de Biophotonique et Pharmacologie UMR 7213 Centre d'Études Internationales et Européennes (CEIE) EA 3396, 228 p.

14. Njassap, N. H. V. (2001). Contribution à l'étude de la qualité microbiologique des produits fermentés Kossam commercialisé dans les rues de Yaoundé (Cameroun). Thèse de Médecine Vétérinaire, Dakar, 230p.

15. OMS. (1998). Réglementation des médicaments à base de plantes, La situation dans le monde. WHO/TRM/98.1, 65 p.

16. OMS. (2000). Principes méthodologiques généraux pour la recherche et l'évaluation relative à la médicine traditionnelle. Genève, Organisation Mondiale de la Santé. 79p

17. OMS. (2002). Stratégie de l'OMS pour la Médecine traditionnelle 2002-2005, Genève, WHO/EDM/TRM/2002. 65p.

18. Sylla, K. S. B. (2000). Contribution à l'étude comparée des conditions de réception de stockage et de préparation des denrées alimentaires d'origine animale dans la Restauration Collective : Cas particulier des Restaurations du Centre des Euvres Universitaires de Dakar (C.O.U.D) -Sénégal, Thèse de Médecine Vétérinaire, Dakar, ${ }^{\circ} 02$, $199 \mathrm{p}$.

19. Pharmacopée européenne. (2014). Qualité microbiologique des préparations pharmaceutiques, pp 567-569.

20. Tayou, F. M. C. (2007). Etude de l'hygiène dans la restauration collective commerciale moderne à Dakar (Sénégal). Thèse de Médecine Vétérinaire de Dakar, Sénégal, 113 p. 